Steffen Ritter

Das Unternehmen Agentur 
Steffen Ritter

\section{Das Unternehmen \\ Agentur}

Erfolgreich selbständig

in der Versicherungswirtschaft

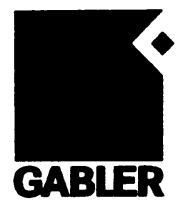


Bibliografische Information Der Deutschen Bibliothek

Die Deutsche Bibliothek verzeichnet diese Publikation in der Deutschen Nationalbibliografie; detaillierte bibliografische Daten sind im Internet über

<http://dnb.ddb.de> abrufbar.

1. Auflage November 2002

Alle Rechte vorbehalten

(C) Betriebswirtschaftlicher Verlag Dr. Th. Gabler GmbH, Wiesbaden 2002

Softcover reprint of the hardcover 1st edition 2002

Lektorat: Susanne Kramer

Der Gabler Verlag ist ein Unternehmen der Fachverlagsgruppe BertelsmannSpringer. www.gabler.de

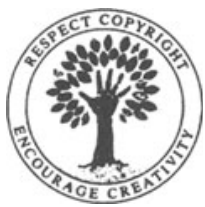

Das Werk einschließlich aller seiner Teile ist urheberrechtlich geschützt. Jede Verwertung außerhalb der engen Grenzen des Urheberrechtsgesetzes ist ohne Zustimmung des Verlags unzulässig und strafbar. Das gilt insbesondere für Vervielfältigungen, Übersetzungen, Mikroverfilmungen und die Einspeicherung und Verarbeitung in elektronischen Systemen.

Die Wiedergabe von Gebrauchsnamen, Handelsnamen, Warenbezeichnungen usw. in diesem Werk berechtigt auch ohne besondere Kennzeichnung nicht zu der Annahme, dass solche Namen im Sinne der Warenzeichen- und Markenschutz-Gesetzgebung als frei zu betrachten wären und daher von jedermann benutzt werden dürften.

Umschlaggestaltung: Nina Faber de.sign, Wiesbaden

ISBN 978-3-322-92092-8

ISBN 978-3-322-92091-1 (eBook)

DOI 10.1007/978-3-322-92091-1 


\section{Vorwort}

Vorab möchte ich Ihnen ganz herzlich danken. Danken für den Erwerb dieses Buches und danken für die Zeit, die Sie in dieses Buch investieren.

„Das Unternehmen Agentur“ wendet sich sowohl an den existenzgründenden Selbständigen in der Versicherungswirtschaft als auch den etablierten Agenturunternehmer, an die betreuende Führungskraft im Vertrieb sowie den Agenturberater. Und selbstverständlich auch an all jene, die kurz vor der Gründung einer Agentur stehen oder mit Agenturunternehmern optimal zusammenarbeiten möchten.

Viele Bücher - zumeist aus dem Bereich der so genannten „Erfolgsliteratur" - enthalten zu Beginn die Aufforderung an den Leser, mit dem Werk zu arbeiten. Die Erfahrungen bestätigen jedoch: Die wenigsten tun es.

Ich möchte Sie darum bitten, den folgenden Seiten gleich zweimal Ihre Zeit zu widmen. Im ersten Schritt einfach lesen, nur lesen! Haben Sie die Gedanken angeregt, selbst zu handeln, kommt erst der zweite Schritt. Nehmen Sie Marker, Bleistift und einige Blatt Papier und arbeiten Sie das Buch nochmals langsam und in Ruhe - eventuell auch in völlig anderer Reihenfolge - durch. Optimal wäre, Sie könnten dies fernab von Ihrem beruflichen Alltag tun.

Kaum eine unternehmerische Entwicklung steht sofort auf der Sonnenseite des Ertrags. Nach meinem eigenen Studium stand für mich auch aufgrund familiärer Prägung - der Weg in die Selbständigkeit fest. In Kombination meines Pädagogikstudiums und meiner betriebswirtschaftlichen Ausbildung gründete ich 1992 mein erstes Unternehmen RITTER.SEMINARE, welches sich ausschließlich der Fortbildung Selbständiger und Unternehmer widmete.

Nicht wenige Monate feilte ich an Seminarkonzeptionen mit dem einzigen Ziel, kaufmännische Fortbildung vor allem verständlich, praxisnah und motivierend zu präsentieren. Im Rückblick auf diese Zeit: Ich kenne Phasen der Investition! Sie sind unvermeidbar und genauso 
bedeutend wie die drei Faktoren, die letztlich den Ausschlag dafür geben, ob Erfolg und Ertrag sich einstellen: Konzentration, Geduld und stets eine klare Ausrichtung.

Neben diesen drei Voraussetzungen liegt der wahrscheinlich wichtigste Baustein solider unternehmerischer Entwicklung ganz woanders. Großer Dank gilt meiner Frau und meinen Kindern für Ihre ständige Unterstützung und vor allem für Thre Geduld. In manch wichtiger Entscheidung waren und sind sie die ,letzte Instanz“. Ebenso danke ich allen Mitarbeitern des Instituts Ritter für die ausgezeichnete Zusammenarbeit und das in den letzten Jahren gemeinsam Erreichte.

Lassen Sie uns nun verschiedene gedankliche Wege zu einem umsatz- und ertragreichen Agenturunternehmen beschreiten. Manch Selbsterkenntnis verursacht gegebenenfalls Schmerzen. Einige der nachfolgenden Gedanken liegen - dessen bin ich mir vollkommen bewusst - keinesfalls im „Trend“.

Aber auch langfristiger Ertrag ist in unserer (konsumverschuldeten) Gesellschaft keinesfalls eine Trenderscheinung. Ein altes japanisches Sprichwort sagt: „Im Trend liegen, heißt am Durchschnitt teilhaben“.

Wenn dem tatsächlich so ist: Sorgen Sie dafür, nicht im Trend zu liegen! Dabei wünsche ich Ihnen viel Erfolg und optimale Erträge!

Sangerhausen, Oktober 2002

Steffen Ritter 


\section{Inhalt}

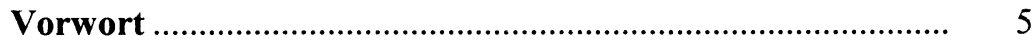

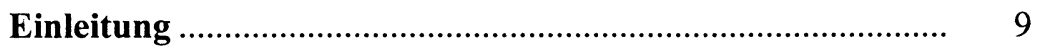

1. Start ins Unternehmertum................................................. 11

1.1 Der Weg zum Unternehmer.................................................. 11

1.2 Auf einmal ist alles anders! ............................................... 15

1.3 Das Fundament der ersten Jahre ......................................... $\quad 20$

1.4 Die Wellen des Unternehmers ............................................... 27

1.5 Konzentration oder Chaos: Papier in der Agentur................. 34

1.6 System ist Pflicht: Geld in der Agentur ................................ 39

1.7 Nicht unmöglich: Wochenplanung in der Agentur................ 44

2. Unternehmensziel Gewinn ................................................. 53

2.1 Strategische Ausgaben einer Agentur................................... 53

2.2 Budgetierung der Agenturausgaben .................................. 59

2.3 Was „bringt“ ein einzelner Kunde? ....................................... 63

2.4 Der Deckungsbeitrag Thres Kunden ..................................... 69

2.5 Der Wert Ihrer Stunden ......................................................... 73

3. Chancen der Gestaltung.................................................... 81

3.1 Agentur und Finanzamt ..................................................... 81

3.2 Die Steuerlast in der Agentur ............................................ 88

3.3 Die „Wahl“ der Gewinnermittlungsvariante......................... 96

3.4 Betriebswirtschaftliche Auswertung einer Agentur.............. 99

3.5 Gewinngestaltung durch Abschreibung............................... 107

3.6 Gewinngestaltung durch Investitionen der Zukunft ............. 115

3.7 Vom Privat- zum Agenturvermögen.................................... 124

3.8 Das Fahrzeug in der Agentur......................................... 127

3.9 Spesen des Agenturinhabers................................................ 133 
4. Investitionen, Personal und Wachstum ............................... 135

4.1 Exkurs in die Kosten....................................................... 135

4.2 Vorbereitende Gedanken zu Ihrer Agenturstrategie .............. 139

4.3 Entscheidung pro Personal? .............................................. 142

4.4 Gezielte Aufgabenteilung in der Agentur............................ 146

4.5 Mitarbeiter kosten Geld!.................................................. 151

4.6 Bringen Sie Ihren Mitarbeiter zum Ertrag! .......................... 155

4.7 Sonderfall „Geringfügige“ in der Agentur .......................... 162

4.8 Das System Threr Agentur ............................................. 165

5. Von der Betriebswirtschaft zum Vertrieb.......................... 170

5.1 Der Mindest-Umsatz in der Agentur ................................. 170

5.2 Der Weg zum Mindest-Umsatz .......................................... 177

5.3 Professionelle Terminierung.............................................. 182

5.4 Vom Markt zum Marketing ............................................ 187

5.5 Das personifizierte Mailing ............................................... 195

5.6 Klassifizierung und Bindung Ihrer Kunden.......................... 201

6. Die Zukunft Ihrer Agentur............................................ 208

6.1 Ab morgen Unternehmer!................................................ 208

6.2 Welche Gewinnentwicklung trauen Sie sich zu? ................ 213

6.3 Entwickeln Sie Thren Wunschbestand!.............................. 222

6.4 Die persönliche Entwicklung des Unternehmers................... 226

6.5 Der Weg zur Vision............................................................. 233

6.6 Von Unternehmertypen zur Unternehmensentwicklung ....... 237

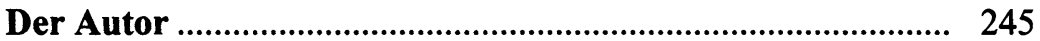

